\title{
Cervical invasion, lymphovascular space invasion, and ovarian metastasis as predictors of lymph node metastasis and poor outcome on stages I to III endometrial cancers: a single-center retrospective study
}

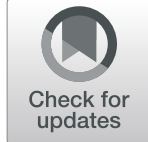

\author{
Min Li', Shuwei Wu, Yangqin Xie, Xiaohui Zhang, Zhanyu Wang, Ying Zhu and Shijie Yan
}

\begin{abstract}
Background: The aim of this study is to determine pathological factors that increase the risk of LNM and indicate poor survival of patients diagnosed with endometrial cancer and treated with surgical staging.

Method: Between January 2010 and November 2018, we enrolled 874 eligible patients who received staging surgery in the First Affiliated Hospital of Anhui Medical University. The roles of prognostic risk factors, such as age, histological subtype, tumor grade, myometrial infiltration, tumor diameter, cervical infiltration, lymphopoiesis space invasion (LVSI), CA125, and ascites, were evaluated. Multivariable logistic regression models were used to identify the predictors of LNM. Kaplan-Meier and COX regression models were utilized to study the overall survival.

Results: Multivariable regression analysis confirmed cervical stromal invasion (OR 3.412, 95\% Cl 1.631-7.141; $P<0.01$ ), LVSI (OR 2.542, 95\% Cl 1.061-6.004; $P=0.04)$ and ovarian metastasis (OR 6.236, 95\% Cl 1.561-24.904; $P=0.01)$ as significant predictors of nodal dissemination. Furthermore, pathological pattern $(P=0.03)$, myometrial invasion (OR 2.70, 95\% Cl 1.139-6.40; $P=0.01$ ), and lymph node metastasis (OR 9.675, 95\% Cl 3.708-25.245; $P<0.01)$ were independent predictors of decreased overall survival.
\end{abstract}

Conclusions: Cervical invasion, lymphopoiesis space invasion, and ovarian metastasis significantly convey the risk of LNM. Pathological type, myometrial invasion, and lymph node metastasis are all important predictors of survival and should be scheduled for completion when possible in the surgical staging procedure.

Keywords: Endometrial cancer, Endometrial carcinoma, Lymph node metastasis, Lymph node dissection, Lymphadenectomy

\section{Background}

Endometrial cancer (EC), the most frequent form of female genital tract tumors [1], lead to approximately $20 \%$ mortality worldwide. Recently, the incidence of endometrial cancer has shown an upward trend year by year [2]. Lymph node metastasis (LNM) is widely recognized as the main prognostic factor for endometrial cancer [3].

So far, routine procedures for EC staging and treatment are total hysterectomy and bilateral salpingo-

\footnotetext{
* Correspondence: liminzhi2009@126.com

Department of Obstetrics and Gynecology, the First Affiliated Hospital of Anhui Medical University, 218 Jixi Road, Hefei 230022, Anhui, China
}

oophorectomy, including pelvic and para-aortic lymphadenectomy [4]. However, whether lymph node dissection (LND) could be applied for endometrial cancer patients remains a controversial issue, either in stage I or in a higher stage $[5,6]$. Many debates about the role of lymphadenectomy exist. According to National Comprehensive Cancer Network (NCCN), surgical staging could be performed in EC patients, and referred to for adjuvant therapy [7]. Wang et al. consider patients with early stage disease should take surgical staging [8]. While some contemporary trials take exception to the effects of lymphadenectomy since it does not prolong overall and 
disease-free survival time $[9,10]$. Therefore, it is necessary to recognize patients who will profit from lymphadenectomy.

One of the challenges in EC is to determine the specific factors that predict lymph node metastasis, so as to optimize the patient group receiving LA and estimate the need for adjuvant therapy. Due to good prognosis of primary endometrial cancer, it is possible and necessary to avoid over treatment, especially for elderly women on whom often increases the risk of complications [11]. Lymphoedema is a well-known long-term complication following LA, and its incidence may increase over time [12]. Much work so far has focused on the relationship between pathological characteristics and lymph node metastasis. There is still no consensus. Therefore, further studies are still required.

In this study, we aim to retrospectively identify pathological factors that increase the risk of LNM and indicate poor survival in patients diagnosed with stages I-III endometrial cancer and treated with surgical staging.

\section{Methods}

Using a retrospective database at the Department of Obstetrics and Gynecology, First Affiliated Hospital of Anhui Medical University, all endometrial cancer patients receiving complete staging surgery between January 2010 and November $2018(n=874)$ were enrolled. The study program was approved by reviewing committee of provincial institutions.

The study population included women with endometrial cancer clinically confined to the uterus who underwent comprehensive surgical staging. Women with high risk factors for nodal metastasis were excluded. These risk factors were as follows: FIGO (International Federation of Gynecology and Obstetrics, 2009) stage IV disease, presence of synchronous carcinoma, experiences of prior neoadjuvant chemotherapy or radiotherapy, along with a secondary malignity. Patients with incomplete data were also excluded from the study. All eligible candidates were divided into "group 1" patients without LNM and "group 2" patients with positive nodes.

Relationship between clinical parameters and lymph node metastasis or overall survival was assessed. Clinical data and tumor characteristics, including age at surgery, histological subtype, tumor grade, depth of myometrial invasion, cervical stromal invasion, peritoneal cytology, primary tumor size (PTD), lymphatic vascular infiltration, and preoperative CA125 level, were extracted from patients' medical records and original pathology reports.

Surgical staging includes total hysterectomy, bilateral salpingectomy, pelvic and aortic lymphadenectomy, and peritoneal irrigation. All patients entering into the study received comprehensive surgical staging-365 (83.5\%) patients with laparotomy while $72(16.5 \%)$ cases of laparoscopy surgery. Pelvic lymph node resection refers to the removal of all lymph nodes, including common ilium lymph nodes, internal ilium lymph nodes, external ilium lymph nodes, and obturator lymph nodes. For grade 3 tumors, paraortic lymph node dissection was performed on high risk patients, those with deep myometrium invasion( $>50 \%$ ), and cervical involvement. Resection of paraortic lymph node involves lymph nodes located at the level of the aortic bifurcation to the renal vein, with the area above the (IMA) of the submesenteric artery contained [13]. All surgical procedures were conducted by gynecological oncologists. The operative data included the total number of LNs resection, the number of pelvic LNs resection, and the number of LNs removal subsequent to the aorta.

All surgical specimens were reviewed and explained by gynecological pathologists. Depending on the World Health Organization classification, the histology classification was carried out [14]. The primary tumor size (PTD), defined as the largest diameter in each of the three dimensions of the tumor, was measured on fresh tissue by pathologists [15]. The overall survival was considered as the time from primary surgery to death or the last follow-up [16]. All tumors were staged according to the FIGO staging system (2009) [17].

The systematic lymph node dissection (LND) is defined as excision of more than 20 nodes [18]. Appropriate pelvic lymphadenectomy is defined as the removal of at least ten pelvic lymph nodes, and appropriate paraaortic lymphadenectomy is defined as the removal of at least five para-aortic lymph nodes [19, 20]. Adjuvant therapies are at the discretion of the therapist, including chemotherapy, radiotherapy, hormone therapy, or some combination of these treatments.

Statistical analysis was performed using Statistical Package Social Sciences (SPSS)23.0. Continuous variables (presented as mean \pm SD or median (range)) were analyzed using the Kolmogorov-Smirnov test to determine whether they were normally distributed. Then Mann-Whitney $U$ test was utilized for non-normal data. As to categorical variable (presented as number and percentage), the chi-square test (Pearson chi-square and Pearson exact chi-square tests) was used to compare the proportions between groups. Univariate and multivariate logistic models were utilized to recognize the risk factors. The Kaplan-Meier method and cox regression were used to evaluate the covariance and generate the survival curves, whose comparisons were made with the log rank test and Breslow (Generalized Wilcoxon) test. A $P$ value $<0.05$ was identified as statistically significant.

\section{Results}

A total of 874 patients met the inclusion criteria of this research and were included. According to the final pathology reports, lymph nodes involvement was found in 82 (9.61\%) patients. Of the 874 patients, 570 (65.22\%) 
presented with FIGO stage I, 212 (24.26\%) with stage II, and $92(10.52 \%)$ with stage III disease. There were 792 in group 1 and 82 in group 2. Baseline clinicopathological characteristics of the cohort were all provided in Table 1. The average age was 53.58 years for all subjects, 53.74 and 51.79 years for group 1 and group 2, respectively. The intermediate number of pelvic lymph nodes harvested was 13 (range, 10-46) in group 1 and 11 (range, $10-33)$ in group 2. $(P=0.17)$. The medium number of para-aortic lymph nodes removed was three (range, $0-23$ ) in group1 and three (range, 0-11) in group $2(P=0.11)$. Among the cohort, $320(36.61 \%)$ had undergone pelvic and para-aortic lymph node dissection and $530(60.64 \%)$ had taken only pelvic lymphadenectomy. Meanwhile, 24 (2.75\%) patients accepted paraaortic lymph node sampling. Univariate comparison between two groups was presented in Table 1.

The variables that showed statistical significance in univariate analysis (shown in Table 1) were included in a multivariate regression analysis. Multivariate comparison between two groups was shown in Table 2. No difference was noted in pathological type, tumor grade, deep myometrial invasion, and preoperative CA125 (see Table 2). Whereas cervical involvement, LVSI, and ovarian metastasis showed statistical significance $(P<0.05)$.

At the endpoint of the observation, a total of 790 (90.39\%) patients survived, and 84 died for unknown reasons. The mean follow-up time was 59.3 months (range, 5-108.5) for all patients, 59.5 months (range, 5-108.5) in group 1, and 56.6 months (range, 8.3-108) for group 2 respectively. In univariate analysis, pathological type $(P<0.01)$, tumor grade $(P<0.01)$, myometrial invasion $(P<0.01)$, cervical involvement $(P<0.01)$, LVSI $(P<0.01)$, lymph node metastasis $(P<$ $0.01)$, and ovarian metastasis $(P<0.01)$ were significantly associated with decreased overall survival. Regression model, as shown in Table 3, emphasized the pathological pattern $(P=$ $0.03)$ and myometrial invasion $(P=0.02)$ as strong predictors of decreased overall survival (OS). In addition, lymph node metastasis $(P<0.01)$ and ovarian involvement $(P=0.01)$ were also confirmed as a potent sign of poor outcome.

Cox curves for OS were plotted for the relationship of survival time with non-endometrioid type, deep myometrial invasion, ovarian metastasis, and positive lymph nodes (Fig. 1). Overall survival significantly decreased in patients with nonendometrioid type, deep myometrial invasion, ovarian and lymph nodes dissemination. (log rank test, $P<0.05$ ). As for adjuvant therapy, Kaplan-Meier plot between two groups was shown in Fig. 2. And the log rank test revealed statistical significance $(P=0.04)$, whereas the multivariate analysis revealed no significance.

\section{Conclusions}

In our study, we demonstrate that the following factors are observed to have separate significance for nodal metastasis: non-endometrioid histology, high tumor grade, deep $(\geq 50 \%)$ myometrial invasion, cervical stromal involvement, lymphatic vascular invasion, ovarian involvement, and CA125. Meanwhile, we confirm that cervical stromal invasion, lymphopoiesis space invasion, and ovarian metastasis are independent predictors of nodal metastasis. Further, non-endometrioid carcinoma, deep myometrial invasion, ovarian metastasis, and lymph node metastasis are independent predictors of decreased survival. Different type of adjuvant therapy has undefined momentous influence on overall survival.

We find cervical invasion in $292(29.80 \%)$ cases, which is in conformity with the range of cervical involvement in other literature [21]. The finding of a significant association between cervical involvement and LNM in this study is in concordance with previous studies [22] and stresses the importance of a thorough preoperative evaluation of cervical invasion. The two clinical studies by Cetinkaya et al. [23] and Boren et al. [22] investigated the relationship of cervical involvement with lymph node dissemination, and conveyed that positive cervical invasion was significantly associated with nodal metastasis in univariate analysis. However, it did not constitute an independent predictor, and led to a different result from our investigation. Previous studies showed that dynamic contrast-enhanced magnetic resonance imaging (CEMRI) was the best tool to assess the cervical involvement [24]. The diagnostic accuracy of CE-MRI was $94.6 \%$, which indicated pathological cervical invasion could be almost excluded in patients diagnosed by CE-MRI as not having cervical invasion [25]. However, the possible reasons for MRI inconsistency with final pathological findings are various [26]. More evidence is needed in order to prove this point.

A study by Akbayir et al. [13] in 2012 revealed that lymphopoiesis space invasion, cervical glandular, and stromal involvement were highly associated with lymph node involvement. Another paper proposed highintermediate risk status and lymphatic vascular space infiltration were useful in assessing risk of pelvic nodal disease, and significantly reduced progression-free and overall survival [16]. More recently, Jorge et al. [27] identified 25,907 patients and confirmed lymphopoiesis space invasion was independently associated with lymph node dissemination and remained an independent predictor of survival. Consistent with the aforementioned researches, we also confirmed that lymphovascular space invasion was an independent predictor of lymph node dissemination.

So far, many researchers have undertaken to recognize the risk factors of nodal dissemination with endometrial carcinoma, and the conclusions are varied. In a nationwide study, the Swedish gynecological cancer group [28] put forward that $\mathrm{MI} \geq 50 \%$, atypia histology, and FIGO 
Table 1 Clinical characteristics of stage I to III endometrial cancer patients stratified by lymph node status

\begin{tabular}{|c|c|c|c|c|}
\hline \multirow[t]{2}{*}{ Characteristics } & \multirow[t]{2}{*}{ Total $N=874$} & \multicolumn{2}{|l|}{$\underline{\text { LNM }}$} & \multirow{2}{*}{$\begin{array}{l}P \\
\text { value }\end{array}$} \\
\hline & & $\begin{array}{l}\text { No } \\
N=792(90.62)\end{array}$ & $\begin{array}{l}\text { Yes } \\
N=82(9.38)\end{array}$ & \\
\hline Age at surgery (years) & $53.58 \pm 0.40$ & $53.74 \pm 0.42$ & $51.79 \pm 1.53$ & 0.24 \\
\hline Pathological type & & & & 0.01 \\
\hline Endometrioid & $760(86.96)$ & $702(88.64)$ & $58(70.73)$ & \\
\hline Non-endometroid & $114(13.04)$ & $90(11.36)$ & $24(29.27)$ & \\
\hline FIGO stage & & & & $<0.01$ \\
\hline 1 & $570(65.22)$ & $570(71.97)$ & 0 & \\
\hline$\|$ & $212(24.26)$ & $212(26.77)$ & 0 & \\
\hline III & $92(10.52)$ & $10(1.26)$ & $82(100)$ & \\
\hline Tumor grade & & & & 0.04 \\
\hline 1 & $240(27.46)$ & $224(28.28)$ & $16(19.51)$ & \\
\hline 2 & $438(50.14)$ & $398(50.25)$ & $34(41.46)$ & \\
\hline 3 & $196(22.40)$ & $170(21.57)$ & $32(39.03)$ & \\
\hline Myometrial invasion & & & & $<0.01$ \\
\hline$<50 \%$ & $586(67.05)$ & $548(69.19)$ & $38(46.34)$ & \\
\hline$\geq 50 \%$ & $288(32.95)$ & $244(30.81)$ & $44(53.66)$ & \\
\hline Primary tumor diameter & & & & 0.26 \\
\hline$<2$ & $100(11.44)$ & $96(12.12)$ & $4(4.88)$ & \\
\hline$\geq 2$ & 774 (88.36) & $696(87.88)$ & $78(95.12)$ & \\
\hline Cervical stromal invasion & & & & $<0.01$ \\
\hline No & $604(69.11)$ & $576(72.73)$ & $28(34.15)$ & \\
\hline Yes & $270(30.89)$ & $216(27.17)$ & $54(65.85)$ & \\
\hline LVSI & & & & $<0.01$ \\
\hline No & $736(84.21)$ & $716(90.40)$ & $48(58.54)$ & \\
\hline Yes & $138(15.79)$ & $76(9.60)$ & $34(41.46)$ & \\
\hline Ascites & & & & 0.49 \\
\hline No & $736(84.21)$ & $670(84.60)$ & $66(80.49)$ & \\
\hline Yes & $138(15.79)$ & $122(15.40)$ & $16(19.51)$ & \\
\hline Ovarian metastasis & & & & $<0.01$ \\
\hline No & $850(97.25)$ & $782(98.74)$ & $68(82.93)$ & \\
\hline Yes & $24(2.75)$ & $10(1.26)$ & $14(17.07)$ & \\
\hline Preoperative CA125 & $24.45(27.70)$ & $23.19(26.52)$ & $41.53(56.46)$ & $<0.01$ \\
\hline Lymphadenectomy & & & & 0.61 \\
\hline Only pelvic & $530(60.64)$ & $478(60.35)$ & $52(63.41)$ & \\
\hline Pelvic + paraaortic & $320(36.61)$ & $294(37.12)$ & $26(31.71)$ & \\
\hline Paraaortic sampling & $24(2.75)$ & $20(2.53)$ & $4(4.88)$ & \\
\hline Median number of LNs removed, $n$ (range) & $13(10-65)$ & $13(10-65)$ & $13(10-33)$ & 0.83 \\
\hline Number of pelvic LNs removed & $13(10-46)$ & $13(10-46)$ & $11(10-33)$ & 0.17 \\
\hline Number of paraoartic LNs removed & $3(0-23)$ & $3(0-23)$ & $3(0-11)$ & 0.11 \\
\hline \multicolumn{5}{|l|}{ Lymph node metastasis } \\
\hline Pelvic LN metastasis only & - & - & $74(90.24)$ & \\
\hline PALN metastasis only & & & 0 & \\
\hline Pelvic and PALN metastasis & & & $8(9.76)$ & \\
\hline
\end{tabular}


Table 1 Clinical characteristics of stage I to III endometrial cancer patients stratified by lymph node status (Continued)

\begin{tabular}{|c|c|c|c|c|}
\hline \multirow[t]{2}{*}{ Characteristics } & \multirow[t]{2}{*}{ Total $N=874$} & \multicolumn{2}{|l|}{$\underline{\text { LNM }}$} & \multirow{2}{*}{$\begin{array}{l}P \\
\text { value }\end{array}$} \\
\hline & & $\begin{array}{l}\text { No } \\
N=792(90.62)\end{array}$ & $\begin{array}{l}\text { Yes } \\
N=82(9.38)\end{array}$ & \\
\hline Status & & & & $<0.01$ \\
\hline Alive & 790 (90.39) & $748(94.44)$ & $42(51.22)$ & \\
\hline Died & $84(9.61)$ & $44(5.56)$ & $40(48.78)$ & \\
\hline Follow-up, month (range) & $59.3(5-108.5)$ & $59.5(5-108.5)$ & $56.6(8.3-108)$ & 0.96 \\
\hline Adjuvant therapy & & & & 0.03 \\
\hline No & 748 (85.58) & $690(87.12)$ & $58(70.73)$ & \\
\hline Chemotherapy only & $94(10.76)$ & $74(9.34)$ & $20(24.39)$ & \\
\hline Radiotherapy only & $28(3.20)$ & $24(3.03)$ & $4(4.88)$ & \\
\hline Both & $4(0.46)$ & $4(0.51)$ & 0 & \\
\hline
\end{tabular}

LVSI lymphovascular space invasion, FIGO international federation of gynecology and obstetrics. Values for continuous variables are mean \pm standard deviation.

Values for categorical variables are number (percentage). LNs lymph nodes, PALN para-aortic lymph node

A $P$ value $<0.05$ was considered to be statistically significant

grade 3 had a strong association with lymph node metastasis. A study by Kadan et al. [29] raised that lower body mass index was a danger factor of nodal metastasis in low-risk endometrial cancer. Wang et al. [8] proposed that CA125 could indicate whether a lymphadenectomy was required. Besides, Lucic et al. [30] considered tumor size $>2 \mathrm{~cm}$, myometrial invasion $\geq 50 \%$, and lymphopoiesis space invasion presented at G2 and G3 had high risk of lymphatic spread. Interestingly, all of the above parameters showed significance only in univariate analysis in this study. The cause of this difference may be the diverse inclusion criteria and deficiency of data. Therefore, additional studies are still essential.

Table 2 Multivariate analysis of factors predictive of lymphatic dissemination using logistic regression models

\begin{tabular}{lll}
\hline Characteristics & \multicolumn{2}{l}{ Multivariate analysis } \\
\cline { 2 - 3 } & OR $(95 \% \mathrm{Cl})$ & $P$ value \\
\hline Pathological type & & - \\
$\quad$ Endometrioid & - & 0.09 \\
$\quad$ Non-endometrioid & $2.107(0.887-5.005)$ & \\
Tumor grade & & - \\
1 & $1.140(0.386-3.370)$ & 0.81 \\
2 & $1.065(0.446-2.546)$ & 0.89 \\
3 & $1.788(0.830-3.851)$ & 0.14 \\
Myometrial invasion & $3.412(1.631-7.141)$ & $<0.01$ \\
Cervical involvement & $2.542(1.061-6.004)$ & 0.04 \\
LVSI & $6.236(1.561-24.906)$ & 0.01 \\
Ovarian metastasis & $0.999(0.997-1.001)$ & 0.28 \\
Preoperative CA125 &
\end{tabular}

$O R$ odds ratio, $C I$ confidence interval, $L V S I$ lymphovascular space invasion A $P$ value of $<0.05$ was considered to be statistically significant Conferent: pathological type (endometrioid); tumor grade (1); myometrial invasion $(<50 \%)$; cervical invasion $(<50 \%)$; LVSI $(-)$; ovarian metastasis $(-)$
In our findings, the absence of skip metastasis (paraaortic positive nodes only) is to some extent in accordance with results from previously published singlecenter investigation [31]. This may mean that pelvic lymph node dissection is possibly an acceptable alternative in selected patients, if surgery or patient-related conditions make it difficult to dissect the aortic artery. Minimally invasive surgery has developed rapidly and can improve the rehabilitation quality of women with endometrial cancer [32]. In our study, $16.5 \%$ patients received laparoscopy. The innovative technology even reduces the risk of surgery in older women, which may expand the scope of lymph node clearance [33]. At the same time, the introduction of sentinel lymph node

Table 3 Multivariate analysis of overall survival in stage I-III endometrial cancer patients

\begin{tabular}{llll}
\hline Covariate & \multicolumn{2}{l}{ Overall survival } & \\
\cline { 2 - 4 } & $95 \% \mathrm{Cl}$ & Hazard ratio & $P$ value \\
\hline Pathological type & & - & 0.03 \\
Endometrioid & - & 2.784 & \\
Non-endometrioid & $1.133-6.839$ & 2.700 & 0.02 \\
Myometrial invasion & $1.139-6.400$ & 8.994 & 0.01 \\
Ovarian metastasis & $1.603-50.477$ & 9.675 & $<0.01$ \\
Lymph node metastasis & $3.708-25.245$ & & 0.18 \\
Adjuvant therapy & & - & 0.06 \\
No & - & 16.792 & 0.03 \\
Chemotherapy & $0.857-329.210$ & 32.350 & 0.15 \\
Radiotherapy & $1.376-760.366$ & 12.024 & \\
Both & $0.422-342.529$ & & \\
\hline
\end{tabular}

$O R$ odds ratio, $C l$ confidence interval, $L V S I$ lymphovascular space invasion A $P$ value of $<0.05$ was considered to be statistically significant Conferent: pathological type (endometrioid); myometrial invasion $(<50 \%)$; ovarian metastasis (-); lymph node metastasis (-); adjuvant therapy (no adjuvant therapy) 


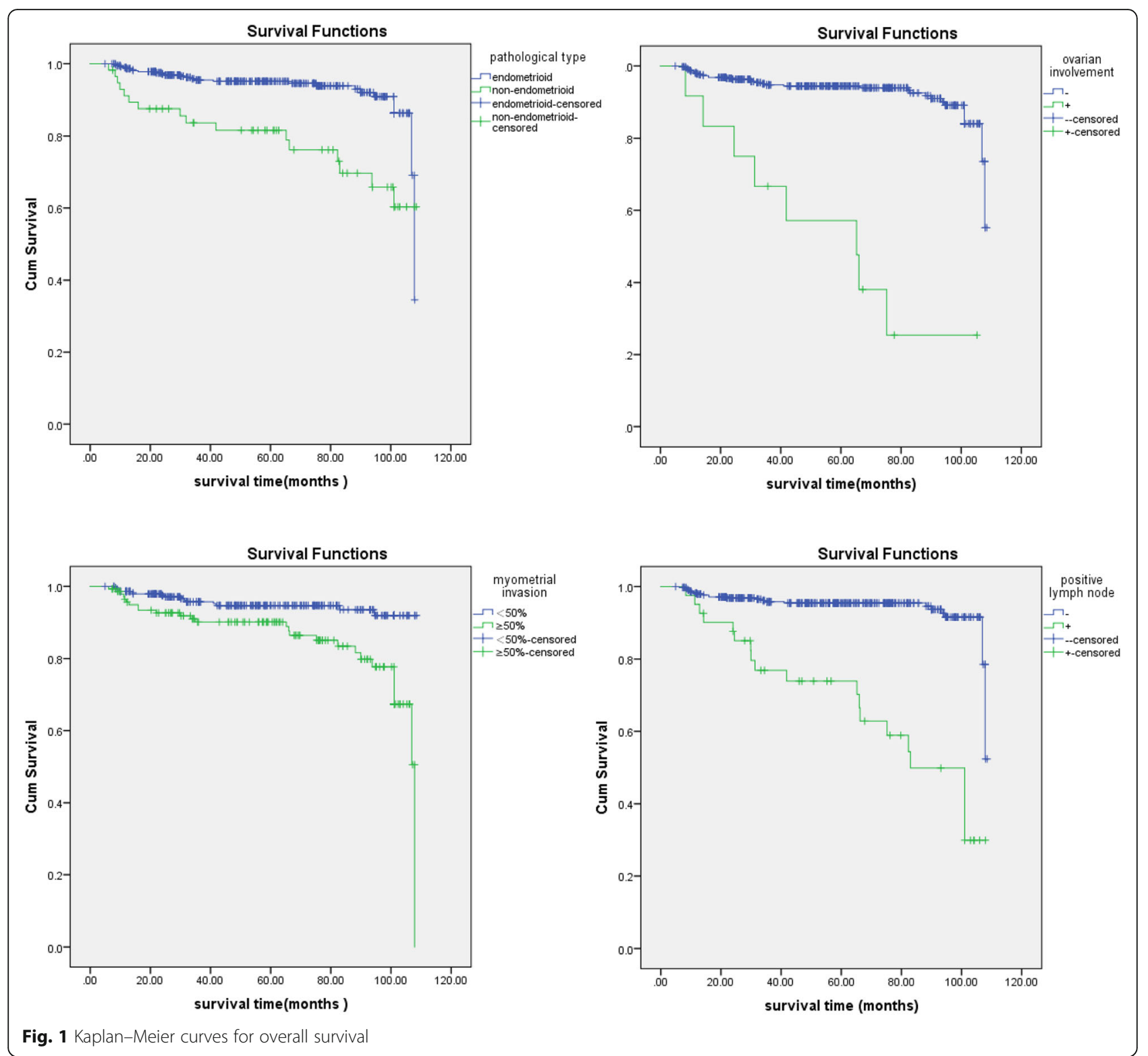

mapping may further reduce surgical trauma. According to NCCN guideline, sentinel lymph node mapping can be regarded as the surgical stage of malignant tumors confined in uterine [7]. Nevertheless, there is no consensus regarding sentinel node mapping in clinical practice in ESGO-ESTRO-ESMO guidelines [34]. In our center, sentinel lymph node mapping is not extensively performed due to no consensus and technical limitations. Therefore, the data are not included in the study.

Poor outcome in endometrial cancer becomes increasingly important in clinical treatment. In our study, positive lymph node and ovarian involvement were related to poor outcome. Meanwhile, histology feature and deep myometrial invasion are strong predictors for diminishing survival. This is in line with
Creutzberg et al. [35] Additionally, postoperative adjuvant therapy does have an effect on the overall survival, although without statistical significance, which is speculated as a result of its low percentage in the total subjects. This conclusion is inconsistent with the results of other contemporary studies [36]. In the PORTEC-1 and PORTEC-2 studies, the effect of EBRT and VRT on patients with high-intermediate risk was observed. Both studies concluded that neither EBRT nor VBT had a positive impact on overall survival compared with the subjects who did not receive adjuvant therapy [37, 38].

The limitations of the study lie in its retrospective nature, with which the accuracy of the results are negatively influenced by recall bias, selected bias, and 


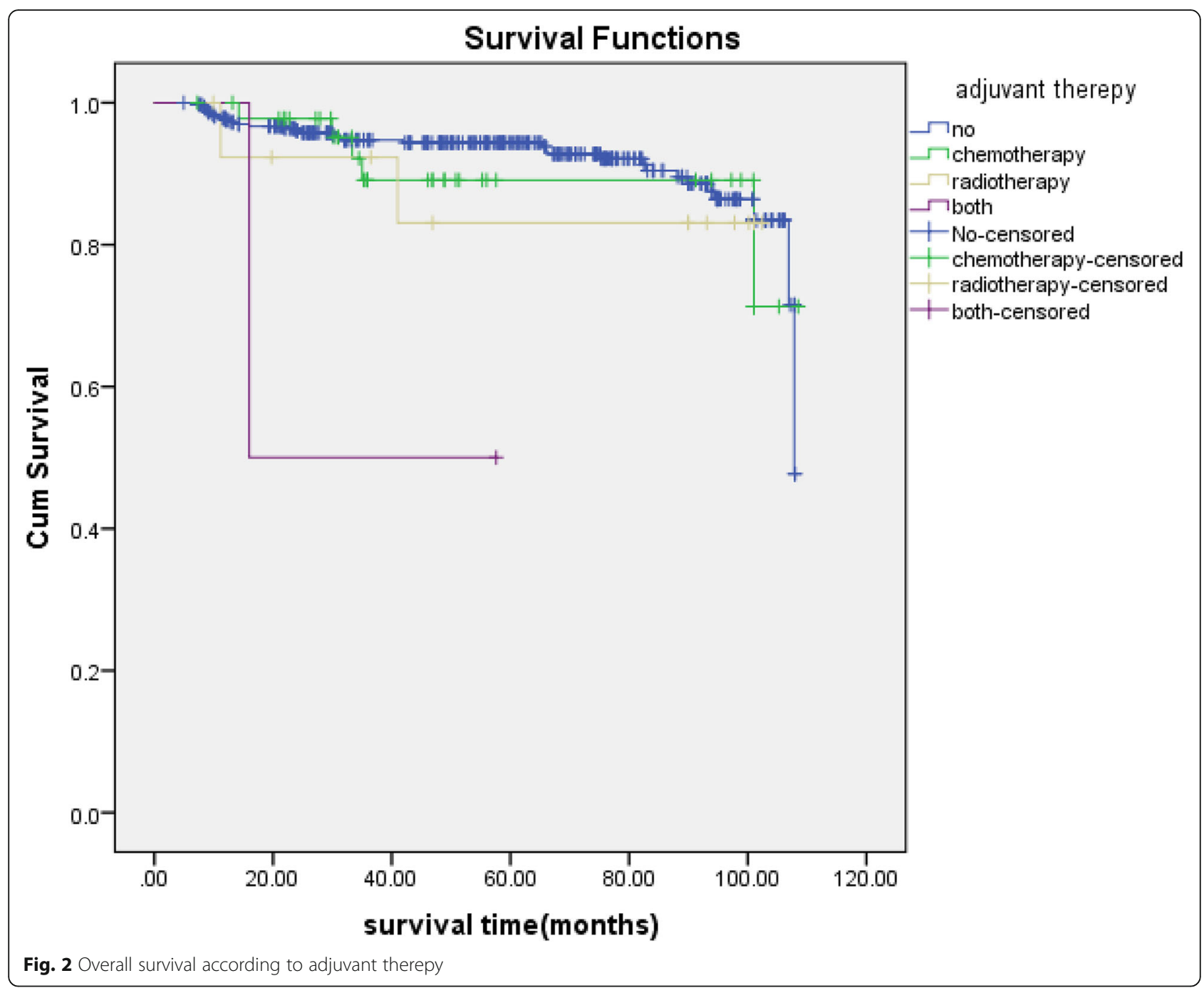

confounding variables [9]. Moreover, during the 8year research period, significant improvements in the clinical techniques also influence the result. In addition, some patients included in the study had incomplete data, which results in errors in the consequences and loss of true statistical significance. Despite these shortcomings, a large number of patients with specific clinical features were enrolled in this study, and the follow-up data available are reliable. Furthermore, as a single-center experience, all invasions treatments were performed by an experienced gynecologic oncologist.

In conclusion, cervical invasion, lymphopoiesis space invasion, and ovarian metastasis dramatically convey the risk of lymph node metastasis. Pathological type, myometrial invasion, and LVSI are all significant predictors of survival and should be completed when possible in the staging of patients with endometrial cancer. Further randomized controlled trials are needed to validate our results.

\section{Abbreviations}

CE-MRI: Contrast-enhanced magnetic resonance imaging; Cl: Confident interval; EC: Endometrial cancer; FIGO: International Federetion of Gynecology and Obstetrics; LNM: Lymph node metastasis;

LVSI: Lymphovascular space invasion; NCCN: National Comprehensive Cancer Network; Od: Odd ratio; OS: Overall survival; PTD: Primary tumor size

\section{Acknowledgments}

Not applicable.

\section{Authors' contributions}

SW analyzed data and drafted the manuscript. YZ and SY participated in data interpretation and manuscript revision. $X Z$ and $Z W$ participated in data acquisition and analysis. ML participated in study concept and design. All authors read and approved the final manuscript.

\section{Funding}

No funding was received.

\section{Availability of data and materials}

The datasets used and/or analyzed during the current study are available from the corresponding author on reasonable request.

\section{Ethics approval and consent to participate}

Due to the retrospective nature of this study, informed consent was waived. But the study was approved by the ethics committee of our hospital. 


\section{Consent for publication}

Consent was obtained from all patients for the publication of the present study。.

\section{Competing interests}

The authors declare that they have no competing interests.

Received: 12 June 2019 Accepted: 23 October 2019

Published online: 16 November 2019

\section{References}

1. Kang S, Nam JH, Bae DS, et al. Preoperative assessment of lymph node metastasis in endometrial cancer: a Korean gynecologic oncology group study. Cancer. 2017;123:263-72.

2. Kathleen M, Brewer Molly A. Endometrial cancer: is this a new disease? Am Soc Clin Oncol Educ Book. 2017;37:435-42.

3. Koskas M, Fournier M, Vanderstraeten A, et al. Evaluation of models to predict lymph node metastasis in endometrial cancer: a multicentre study. Eur J Cancer. 2016;61:52-60.

4. Morice P, Leary A, Creutzberg C, et al. Endometrial cancer. Lancet. 2016;387: 1094-108.

5. Arif K, Emel K, Handan C, et al. Is surgical staging necessary for patients with low-risk endometrial cancer? A retrospective clinical analysis. Asian Pac J Cancer Prev. 2015;16:5331-5.

6. Teixeira AM, Ribeiro R, Schmeler KM, et al. A preoperative and intraoperative scoring system to predict nodal metastasis in endometrial cancer. Int J Gynaecol Obstet. 2017;137:78-85.

7. Wui-Jin K, Abu-Rustum Nadeem R, Sarah B, et al. Uterine Neoplasms, Version 1.2018, NCCN Clinical Practice Guidelines in Oncology. J Natl Compr Cancer Netw. 2018;16:170-99.

8. Wang $\mathrm{Y}$, Han $\mathrm{C}$, Teng F, et al. Predictive value of serum HE4 and CA125 concentrations for lymphatic metastasis of endometrial cancer. Int J Gynaecol Obstet. 2017;136:58-63.

9. Solmaz U, Mat E, Dereli M, et al. Lymphovascular space invasion and cervical stromal invasion are independent risk factors for nodal metastasis in endometrioid endometrial cancer. Aust N Z J Obstet Gynaecol. 2015:55:81-6.

10. ASTEC Study Group, Kitchener H, Swart AM, et al. Efficacy of systematic pelvic lymphadenectomy in endometrial cancer (MRC ASTEC trial): a randomized study. Lancet. 2009;373:125-36.

11. Tanja S, Tone B, Hanno U, et al. Metabolic risk score and cancer risk: pooled analysis of seven cohorts. Int J Epidemiol. 2015;44:1353-63.

12. Carlson JW, Kauderer J, Hutson A, et al. GOG 244, the lymphedema and gynecologic cancer (LEG) study: incidence and risk factors in newly diagnosed patients. Gynecol Oncol. 2018;149:6-7.

13. Akbayir $\mathrm{O}$, Corbacioglu A, Goksedef BP, et al. The novel criteria for predicting pelvic lymph node metastasis in endometrioid adenocarcinoma of endometrium. Gynecol Oncol. 2012;125:400-3.

14. Creasman WT, Odicino F, Maisonneuve P, et al. Caicinoma of the corpus uteri, FIGO 26th Annual Report on the Results of Treatment in Gynecological Cancer. Int J Gynaecol Obstet. 2016;95:105-43.

15. Mariani A, Webb MJ, Keeney GL, et al. Low-risk corpus cancer: is lymphadenectomy or radiotherapy necessary?.Am. J Obstet Gynecol. 2000; 182:1506-19.

16. Guntupalli SR, Zighelboim I, Kizer NT, et al. Lymphovascular space invasion is an independent risk factor for nodal disease and poor outcomes in endometrioid endometrial cancer. Gynecol Oncol. 2012;124:31-5.

17. Pecorelli S. Revised FIGO staging for carcinoma of the vulva, cervix, and endometrium. Int J Gynecol Obstet. 2009;105:103-4.

18. Thomas MB, Mariani A, Cliby WA, et al. Role of systematic lymphadenectomy and adjuvant therapy in stage I uterine papillary serous carcinoma. Gynecol Oncol. 2007;107:186-9.

19. Mariani A, Webb MJ, Keeney GL, et al. Low-risk corpus cancer: is lymphadenectomy or radiotherapy necessary? Am J Obstet Gynecol. 2000; 182:1506-19.

20. Nomura H, Aoki D, Suzuki N, et al. Analysis of clinicopathologic factors predicting para-aortic lymph node metastasis in endometrial cancer. Int J Gynecol Cancer. 2006;16:799-804.

21. Boren T, Lea J, Kehoe S, et al. Lymph node metastasis in endometrioid adenocarcinomas of the uterine corpus with occult cervical involvement. Gynecol Oncol. 2012;127:43-6.
22. Shah C, et al. Does size matter? Tumor size and morphology as predictors of nodal status and recurrence in endometrial cancer. Gynecol Oncol. 2005: 99:564-70.

23. Cetinkaya K, Atalay F, Bacinoglu A. Risk factors of lymph node metastases with endometrial carcinoma. Asian Pac. J. Cancer Prev. 2014;15:6353-6.

24. Sala E, Rockall A, Kubik-Huch RA. Advances in magnetic resonance imaging of endometrial cancer. Eur Radiol. 2011;21:468-73.

25. Fei T, Yan-Fang Z, Wang Y-M, et al. Contrast-enhanced MRI in preoperative assessment of myometrial and cervical invasion, and lymph node metastasis: diagnostic value and error analysis in endometrial carcinoma. Acta Obstet Gynecol Scand. 2015;94:266-73.

26. Yamashita Y, Mizutani H, Torashima M, et al. Assessment of myometrial invasion by endometrial carcinoma: transvaginal sonography vs contrastenhanced MR imaging. AJR Am J Roentgenol. 1993;161:595-9.

27. Jorge $\mathrm{S}$, Hou JY, Tergas Al, et al. Magnitude of risk for nodal metastasis associated with lymphvascular space invasion for endometrial cancer. Gynecol Oncol. 2016;140:387-93.

28. Stålberg K, Kjølhede P, Bjurberg M, et al. Risk factors for lymph node metastases in women with endometrial cancer: a population-based, nationwide register study-on behalf of the Swedish gynecological cancer group. Int J Cancer. 2017;140:2693-700.

29. Kadan Y, Calvino AS, Katz A, et al. Predictors for lymph nodes involvement in low risk endometrial cancer. J Obstet Gynaecol. 2017;37:514-8.

30. Lucic N, Draganovic D, Sibincic S, et al. Myometrium invasion, tumour size and lymphovascular invasion as a prognostic factor in dissemination of pelvic lymphatics at endometrial carcinoma. Med Arch. 2017;71:325-9.

31. Kumar S, Podratz KC, Bakkum-Gamez JN, et al. Prospective assessment of the prevalence of pelvic, paraaortic and high paraaortic lymph node metastasis in endometrial cancer. Gynecol Oncol. 2014;132:38-43.

32. Christer B, Grigorios K, Asciutto Katrin C, et al. A population-based registry study evaluating surgery in newly diagnosed uterine cancer. Acta Obstet Gynecol Scand. 2016;95:901-11.

33. Bourgin C, Lambaudie E, Houvenaeghel $G$, et al. Impact of age on surgical staging and approaches (laparotomy, laparoscopy and robotic surgery) in endometrial cancer management. Eur J Surg Oncol. 2017;43:703-9.

34. Nicoletta C, Carien C, Frederic A, et al. ESMO-ESGO-ESTRO Consensus Conference on Endometrial Cancer: diagnosis, treatment and follow-up. Int. J Gynecol Cancer. 2016;26:2-30.

35. Creutzberg CL, van Putten WL, Koper PC, et al. Surgery and postoperative radiotherapy versus surgery alone for patients with stage 1 endometrial carcinoma: multicenter randomized trial. PORTEC study group. Post operative radiation therapy in endometrial cancer. Lancet. 2000;355:1404-11.

36. Hogberg T, Signorelli M, de Oliveira CF, et al. Sequential adjuvant chemotherapy and radiotherapy in endometrial cancer-results form two randomised studies. Eur J Cancer. 2010;46:2422-31.

37. Creutzberg CL, van Putten WL, Koper PC, et al. Surgery and postoperative radiotherapy versus surgery alone for patients with stage-1 endometrial carcinoma: multicentre randomised trial. PORTEC study group. Post operative radiation therapy in endometrial carcinoma. Lancet. 2000;355: 1404-11.

38. Nout RA, Smit VTHBM, Putter $\mathrm{H}$, et al. Vaginal brachytherapy versus pelvic external beam radiotherapy for patients with endometrial cancer of highintermediate risk (PORTEC-2): an open-label, non-inferiority, randomised trial. Lancet. 2010;375:816-23.

\section{Publisher's Note}

Springer Nature remains neutral with regard to jurisdictional claims in published maps and institutional affiliations. 